\title{
The isophote maps of the Gegenschein obtained by CCD observations
}

\author{
Masateru Ishiguro ${ }^{1}$, Hideo Fukushima ${ }^{2}$, Daisuke Kinoshita ${ }^{3}$, Tadashi Mukai ${ }^{1}$, Ryosuke Nakamura ${ }^{4}$, \\ Jun-ichi Watanabe ${ }^{2}$, Takeshi Watanabe ${ }^{1}$, and John F. James ${ }^{5}$ \\ ${ }^{1}$ The Graduate School of Science and Technology, Kobe University, Kobe 657-8501, Japan \\ ${ }^{2}$ National Astronomical Observatory, Mitaka, Tokyo 181-8588, Japan \\ ${ }^{3}$ Department of Physics, Faculty of Science, Tohoku University, Sendai 980-77, Japan \\ ${ }^{4}$ Information Processing Center, Kobe University, Nada, Kobe 657-8501, Japan \\ ${ }^{5} 29$, Merchants' Quay, Salford, M5 2XF, England
}

(Received December 8, 1997; Revised January 16, 1998; Accepted February 3, 1998)

\begin{abstract}
We summarize the results of our Gegenschein observations at Norikura (altitude 2876 m, Japan) in Sept. 18, 1996, and at Kiso (altitude 1130 m, Japan) in Feb. 9-14, and March 4 and 5,1997. The instrument consisting of fish-eye lens or the wide angle lens attached to the cooled CCD camera was used with green filter. It is found that the position of the maximum brightness near the antisolar point (the Gegenschein) is slightly deviated approximately $0 . .^{\circ} 4$ from the antisolar point to the south in September, whereas nearly $0 .^{\circ} 7$ to the north in February/March. Furthermore, a gradient of the relative intensity along a line perpendicular to the ecliptic is remarkably different with changing seasons, i.e. the brightness decreases faster towards the north in September, in contrast with its quick decrease towards the south in February/March. Our observed evidence suggests that these variations of the peak position and the brightness gradient of the Gegenschein are caused by the annual motion of the Earth with respect to the plane of the zodiacal cloud, and does not conflict with the predictions deduced from the cloud model having its symmetric plane beyond the Earth's orbit coinciding with the invariable plane of the solar system.
\end{abstract}

\section{Introduction}

The Gegenschein is an extremely faint light around the antisolar point. Such faint excess of light was explained by many hypotheses (see, e.g. the review by Roosen, 1971), such as the reflected sunlight by particles concentrated near the libration point in the Earth-Moon system, the emission from the Earth's gaseous tail, the scattered sunlight by Earth's dust tail cloud, and the scattered sunlight by the interplanetary dust cloud. Since the Gegenschein is still present in Pioneer 10 data out to $1.86 \mathrm{AU}$ (Weinberg et al., 1973), it is widely accepted that the Gegenschein is the enhancement of the scattered sunlight in the back-ward direction by the interplanetary dust grains. However there still remain some questions about the Gegenschein.

The first question is its exact position of the maximum brightness. Tanabe (1965) reported that the position of the photometric center of the Gegenschein moved periodically to both sides of the ecliptic; to the north in spring and to the south in autumn. Roosen (1970) and Leinert (1975) cited that Dumont (1965) reported the maximum brightness coincided with the antisolar point. However, as a reviewer of the manuscript, Dumont makes the comment that "Despite their poorer definition (the netmesh of $4^{\circ}$ ), my Gegenschein isophotes (Fig. VI-4, p. 306 in Dumont (1965)), observed in February, are in good agreement with the present isophotes shown in Fig. 2, because i) they exhibit a northward shift $\left(2^{\circ}\right.$ or $3^{\circ}$ ) of the brightness peak along scans perpendicular to the ecliptic at longitudes $\pm 5^{\circ}$ to $\pm 10^{\circ}$ right and left of the anti-

Copy right (C) The Society of Geomagnetism and Earth, Planetary and Space Sciences (SGEPSS); The Seismological Society of Japan; The Volcanological Society of Japan; The Geodetic Society of Japan; The Japanese Society for Planetary Sciences. sun, and ii) as approaching the antisolar longitude, this shift decreases and almost vanishes, a trend which also appears in both Figs. 1 and 2 of the present work".

The second question is the value of its excess brightness. When we observe the Gegenschein, we measure the total brightness coming from the different sources, i.e. the scattered light from urban lightning (the city light), the airglow emission, the scattered sunlight by lower atmosphere of the Earth, the light of background stars (integrated starlight), as well as the Gegenschein. Leinert (1975) reported the maximum brightness of the Gegenschein is about $200 \mathrm{~S}_{10}$ and its excess brightness is about $40 \mathrm{~S}_{10}$, where the equivalent of $1 \mathrm{~S}_{10}$ at a wavelength of $530 \mathrm{~nm}$ is $1.20 \times 10^{-9} \mathrm{erg}$. $\mathrm{cm}^{-2} \mathrm{~s}^{-1}$ ster $^{-1} \AA^{-1}$. Subtraction of other elements to get the excess brightness of the Gegenschein alone is still very difficult task.

The third question is its shape. In general the shape of the Gegenschein is oval elongating east-west direction (e.g. Roosen, 1971). It was difficult to define its precise shape photometrically because there was no available detector for mapping the faint diffuse object, such as the Gegenschein simultaneously. However, it is getting easier recently to take an isophote map of the Gegenschein by using the cooled CCD camera attached to the fish-eye lens, as we used here.

Main purpose of our observations presented here is to measure the shape of the Gegenschein by using the cooled CCD camera, and subsequently to estimate the position of its maximum brightness, and also to monitor their variations with the season. It is rather difficult to obtain the absolute brightness of the Gegenschein, because of the inevitable dark noise in CCD. We checked the dark noise in the evening and after 


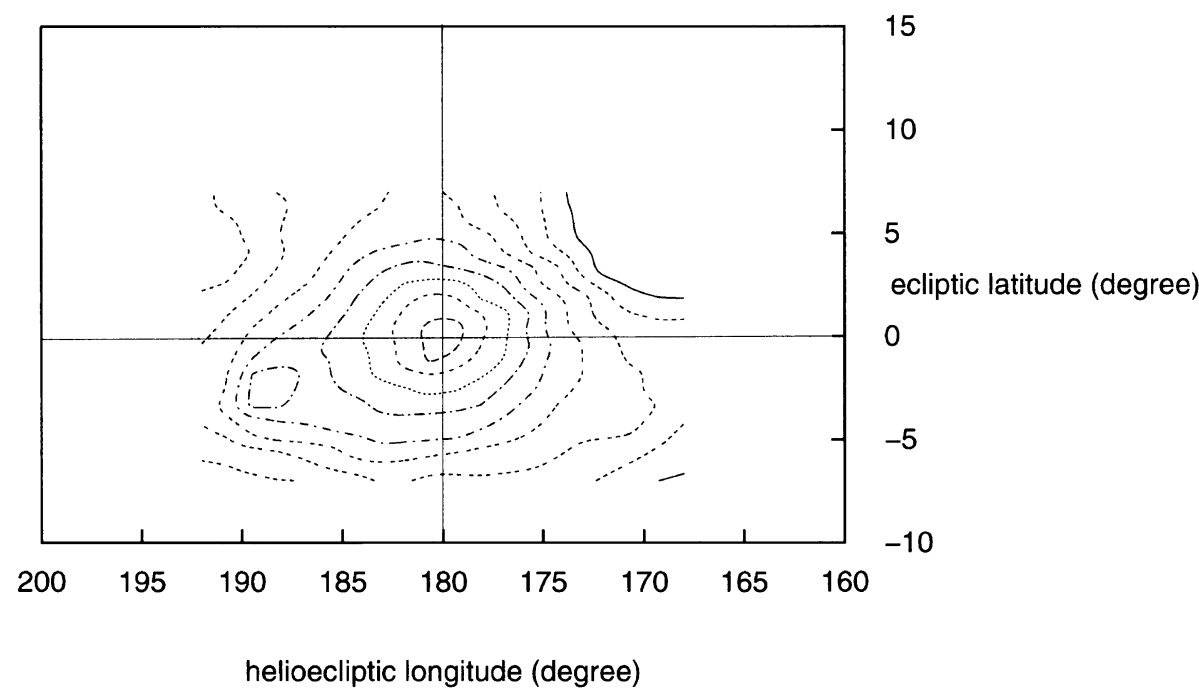

Fig. 1. The isophote map, in arbitrary units, of the Gegenschein in Sept. 18, 1996 at Norikura. A slight hump in the left-down side is a residue of the effect of Jupiter due to incomplete deletion.

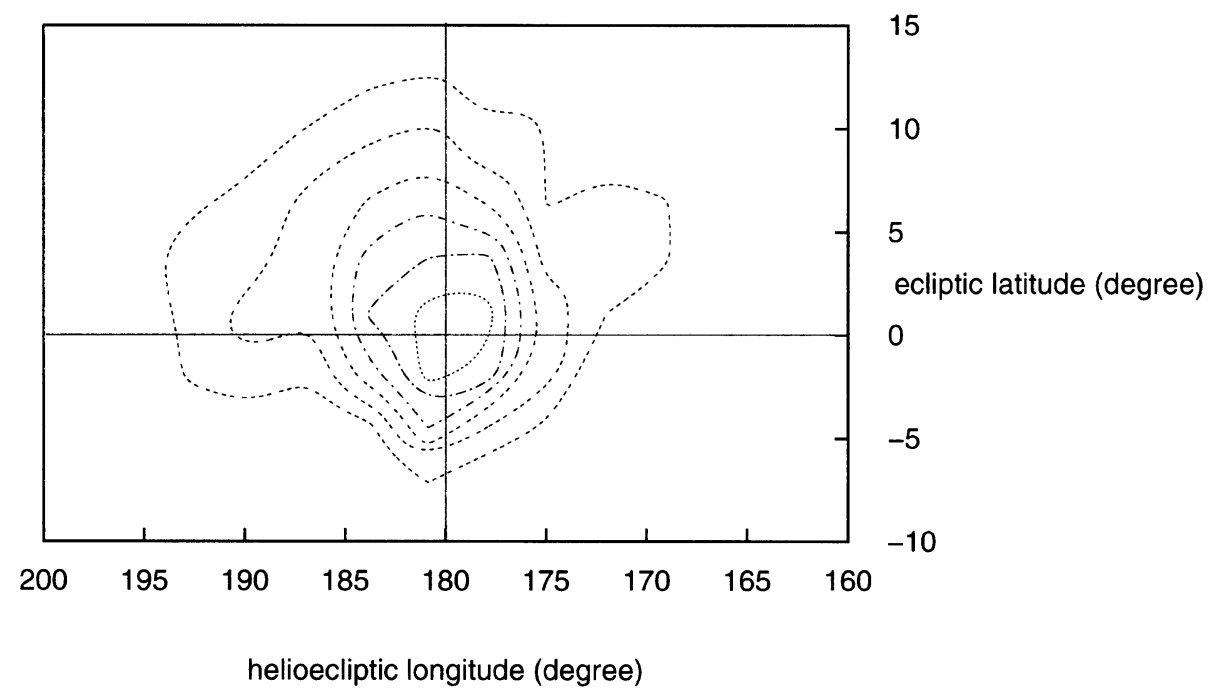

Fig. 2. The isophote map, in arbitrary units, of the Gegenschein in March 5, 1997 at Kiso.

the dawn every day, and found that the efficiency of the refrigerater was not stable. Since a slight variation of the dark noise might be included in the data, we will not discuss here the absolute brightness of the Gegenschein.

\section{Observations}

The photometric data presented here consist of three different observations; at Norikura (2876 m, Nagano, Japan) in September, 1996 and Kiso (1130 m, Nagano, Japan) in February and March, 1997. The details of each observation are as follows.

\subsection{Norikura}

Exposures were made in the 18th September, 1996 at the Norikura Observatory (altitude $2876 \mathrm{~m}$, latitude $36^{\circ} 6.8^{\prime} \mathrm{N}$ ), which is one branch of the National Astronomical Observatory, Japan. We used the instrument consisting of the $20 \mathrm{~mm}$ F5.6 wide angle lens attached to the Mutoh CV-16 CCD cam- era. The number of pixels of Mutoh CV-16 CCD camera is $1536 \times 1024$ pixels, and the pixel spacing is $9 \mu \mathrm{m} \times 9 \mu \mathrm{m}$. Since the Gegenschein is very faint object, we set up $2 \times 2$ binning. As the result, the spatial resolution becomes 20 pixel. $\mathrm{deg}^{-1}$ and the field of view is $38^{\circ} \times 25^{\circ}$. The exposure time was selected as $20 \mathrm{~min}$ by using the equatorial. The filter of Hoya G-533 was applied, which roughly corresponds to the standard V band filter. Since the sky was very fine and clear in the 18th September, 1996, we could get the significant data by one night observation alone.

The zenith angle $Z$ of the Gegenschein at midnight was $38 \mathrm{deg}$ in Sept. 18, 1996. Since the large cities of Nagoya and Gifu are located in the direction of south-west, the contamination of the city light in the south-west sky appeared. Therefore, the data in the lower sky than $Z \geq 45$ deg were discarded. As a result, the available data cover the region of sky, $167^{\circ} \leq \lambda-\lambda_{\odot} \leq 193^{\circ},-7^{\circ} \leq \beta \leq 7^{\circ}$, where $\lambda-\lambda_{\odot}$ 
Table 1. The position of the maximum brightness deduced from CCD observations near the antisolar point (the Gegenschein).

\begin{tabular}{cccc}
\hline Site & Date & North-South deviation (deg.) & East-West deviation (deg.) \\
\hline Haleakala $^{(1)}$ & 1995.9 .22 & -2.5 & 0 \\
Norikura $^{(2)}$ & 1996.9 .18 & -0.4 & 0 \\
Kiso $^{(2)}$ & 1997.3 .5 & +0.7 & 0 \\
\hline
\end{tabular}

(1) James et al. (1997), (2) this paper.

denotes the differential helioecliptic longitude and $\beta$ means the ecliptical latitude.

\subsection{Kiso}

At Kiso Observatory (altitude $1130 \mathrm{~m}$, latitude $35^{\circ} 47.6^{\prime} \mathrm{N}$ ), we observed from the 9th to the 14th February, 1997 and in the 4 th and the 5 th March, 1997. We used a $8 \mathrm{~mm}$ Nikon fisheye lens to get widespread image of the Gegenschein. The CCD camera used here is the Mutoh CV-16 CCD camera, which is the same one as we used at Norikura. We set up $2 \times 2$ binning, and subsequently the spatial resolution became 7.8 pixel. $\mathrm{deg}^{-1}$ and the field of view was $98^{\circ} \times 66^{\circ}$. We selected the exposure time as $20 \mathrm{~min}$, and applied the filters of Hoya G533 and Schott B. Unfortunately, we found that the exposure time in the case of Schott B filter was not enough to reduce the significant data. Therefore, we concentrated our analyses only on the data obtained by using the HOYA G-533 filter.

In this season, the position of the Gegenschein at midnight is near the place of the zenith angle $Z \sim 20 \mathrm{deg}$. Consequently, the atmospheric extinction and the contamination by the airglow can be minimized. Moreover as the temperature of the open air was below $-10^{\circ} \mathrm{C}$ at Kiso, we could keep by the refrigerater the favourable temperature of $-30^{\circ} \mathrm{C}$ to reduce the dark noise largely on the CCD tip.

Both Kiso Observatory and Norikura Observatory are located in the direction of about $100 \mathrm{~km}$ and $120 \mathrm{~km}$, respectively, north-east of Nagoya city. Therefore the similar contamination of city light in the low south-west sky, as noted in the observation at Norikura, appeared in the observation at Kiso. Furthermore we occasionally had a heavy snow in Kiso in February, 1997, and consequently the reflected light from the ground was not negligibly small. Considering the contamination due to the city light and the weather condition, we found that the data obtained in the 5th March, 1997 are most reliable, and the available data for analysis cover $165^{\circ} \leq \lambda-\lambda_{\odot} \leq 195^{\circ},-10^{\circ} \leq \beta \leq 15^{\circ}$.

\section{Data Reduction}

In the ground observations, the observed intensity $\left(I_{\mathrm{obs}}\right)$ of the night sky is composed of those of starlight $\left(I_{\mathrm{ST}}\right)$, zodiacal $\operatorname{light}\left(I_{\mathrm{ZL}}\right)$, airglow $\left(I_{\mathrm{AG}}\right)$, scattered light by lower atmosphere $\left(I_{\mathrm{SL}}\right)$ and the city light $\left(I_{\mathrm{CL}}\right)$.

$$
I_{\mathrm{obs}}=\left(I_{\mathrm{ST}}+I_{\mathrm{ZL}}+I_{\mathrm{AG}}\right) \cdot e^{-\tau}+I_{\mathrm{SL}}+I_{\mathrm{CL}},
$$

where $\tau$ denotes the optical depth of the atmosphere. We estimated $\tau \sim 0.10$ from the observations of a zenith angle $Z$ variation of the brightness of the reference stars. Especially main contamination in $\mathrm{G}$ band is OI airglow emission $(557.7 \mathrm{~nm})$. Van Rhijn (1919) reported that this airglow has almost the uniform density layer. Therefore, we can approximate the brightness of airglow as a function of the zenith angle $Z$.

The first step of the data reduction is flattening of the image frame. Fish-eye lens has its peculiar property, that is, the detected intensity in the center part of the frame is brighter than that in the edge of frame. In order to remove this effect, the raw data were divided by those detected for the flat field frame, which was acquired by taking the images of the fish tank filled with water and milk (a volume ratio of two components is about 10:1) as the similar method to that described in James et al. (1997). After this reduction, we removed the stars by using the computer program and smoothed the results. Almost all stars brighter than $~ 9$ mag can be removed, except some bright objects such as planets of Jupiter, Saturn and Mars. The areas of CCD influenced by these bright objects, as well as by the background field stars in the Milky Way were smoothed computationally. A contribution from the stars fainter than $\sim 9$ mag is sufficiently low, i.e. below the noise caused by dark current.

The residual components of contamination are those due to airglow and city light. To determine the Van Rhijn function, we fit the minimum values of each zenith distance to arbitrary exponential function, and subtract the smoothed data by its function in order to find out the enhanced region by back scattering. The isophote maps of the reduced data are shown in Fig. 1 for that obtained at Norikura, and in Fig. 2 at Kiso.

\section{Results}

It is clear that the shape of the Gegenschein changes with the season (see Figs. 1 and 2). In addition, a center position of the maximum brightness of the Gegenschein deviates to the south in September and to the north in March. We have estimated the amount of the deviation, i.e. the latitude of the Gegenschein becomes about $-0 .^{\circ} 4$ in September 1996 and $+0 .^{\circ} 7$ in March 1997, while its longitude is nearly the same as the anti-solar point (see Table 1). For reference, the result obtained by the cooled CCD camera at Haleakala (altitude 3000 m, Hawaii, USA) (James et al., 1997), -2. ${ }^{\circ} 5$ in September 1995 is also listed in Table 1. Observations of the latitude of the Gegenschein by various photoelectric and photographic observers compiled by Roosen (1970) have been shown in Fig. 3, including three data listed in Table 1.

The north-south deviation found in the observations can be explained by the zodiacal cloud model having a symmetric plane deviated toward the south in September and toward the north in March (see, e.g. Misconi, 1981). The invariant plane of the solar system is one plausible candidate, as shown in 


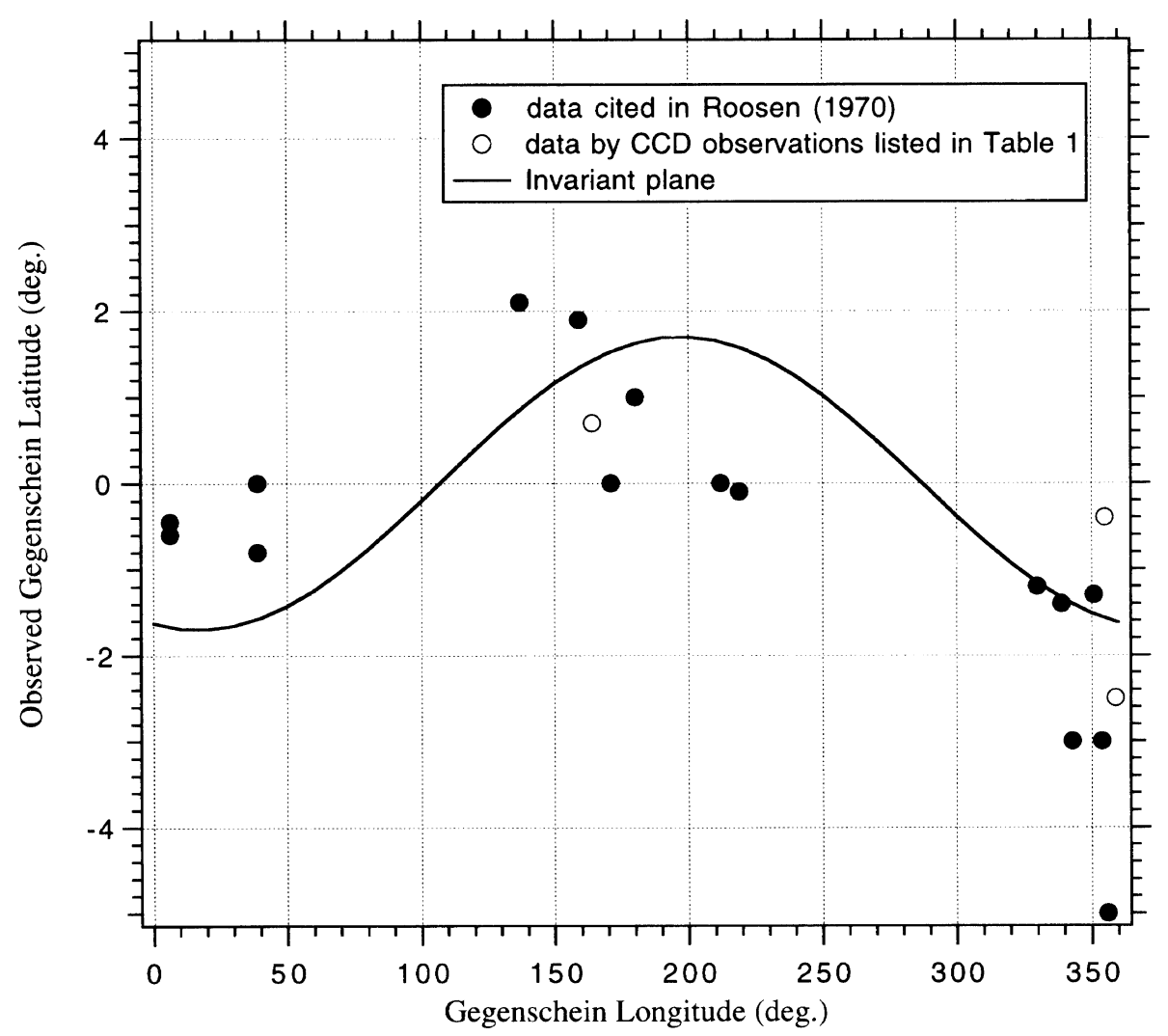

Fig. 3. Observed position of the maximum intensity of the excess brightness (the Gegenschein) near the antisolar point.

Fig. 3, but we need more data to discuss a time variation of such daviation.

It has been proposed by Hong and Kwon (1991) that if the observed resolution of the Gegenschein is better that $\sim 3^{\circ}$, one could show to be different in the cloud models. Since our observations have provided the isophote maps with the resolution better than $0 .^{\circ} 5$, they are available to compare with those predicted by the several cloud models to estimate the structure of the zodiacal cloud.

We could not estimate the absolute brightness because of unremovable contamination by dark noise in CCD and OI airglow. By using the filters which can exclude the airglow, as well as more sensitive CCD camera, we will obtain the information of absolute brightness in our future observations.

Acknowledgments. We thank the members of the Norikura Observatory (National Astronomical Observatory, Japan) and the Kiso Observatory (University of Tokyo, Japan) for their kind helps during the observations. We also thank the useful review works by Drs. R. Dumont and N. Misconi.

\section{References}

Dumont, R., Separation of the atmospheric, interplanetary and stellar components of the night sky at $5000 \mathrm{~A}$ : Application to the photometry of the zodiacal light and the gegenschein (in French), Ann. Astrophys., 28, 265-320, 1965 (English Transl., NASA TT F11, 164, 1965).

Hong, S. S. and S. M. Kwon, On the gegenschein and the symmetry plane, in Origin and Evolution of Interplanetary Dust, edited by A. C. LevasseurRegourd and H. Hasegawa, pp. 147-150, Kluwer Academic Publ., 1991.

James, J. F., T. Mukai, T. Watanabe, M. Ishiguro, and R. Nakamura, The morphology and brightness of the zodiacal light and gegenschein, Mon. Not. R. Astron. Soc., 288, 1022-1026, 1997.

Leinert, C., Zodiacal light-A measure of the interplanetary environment, Space Sci. Rev., 18, 281-339, 1975.

Misconi, N. Y., The photometric center of the gegenschein, Icarus, 47, 265-269, 1981.

Roosen, R. G., An annotated bibliography on the gegenschein, 1970, Icarus, 13, 523-539, 1970.

Roosen, R. G., The gegenschein, Rev. of Geophys. Space Phys., 9, 275-304, 1971.

Tanabe, H., Photoelectric observations of the gegenschein, Publ. Astron. Soc. Japan, 17, 339-366, 1965.

Van Rhijn, P. J., On the brightness of the sky at night and the total amount of starlight, Astrophys. J., 50, 356-375, 1919.

Weinberg, J. L., M. S. Hanner, H. M. Mann, P. B. Hutchison, and R. Fimmel, Observation of zodiacal light from the Pioneer 10 asteroid-Jupitor probe, Space Research, XIII, 1187-1193, 1973.

M. Ishiguro (e-mail: ishiguro@komadori.planet.kobe-u.ac.jp), H. Fukushima, D. Kinoshita, T. Mukai, R. Nakamura, J. Watanabe, T. Watanabe, and J. F. James 